University of Wollongong

Research Online

Faculty of Engineering - Papers (Archive)

Faculty of Engineering and Information

Sciences

$1-1-2005$

\title{
Spatial rotation kinematics and flexural-torsional buckling
}

Lip H. Teh

University of Wollongong, Iteh@uow.edu.au

Follow this and additional works at: https://ro.uow.edu.au/engpapers

Part of the Engineering Commons

https://ro.uow.edu.au/engpapers/632

\section{Recommended Citation}

Teh, Lip H.: Spatial rotation kinematics and flexural-torsional buckling 2005, 598-605.

https://ro.uow.edu.au/engpapers/632

Research Online is the open access institutional repository for the University of Wollongong. For further information contact the UOW Library: research-pubs@uow.edu.au 


\title{
SPATIAL ROTATION KINEMATICS AND FLEXURAL- TORSIONAL BUCKLING
}

\author{
By Lip H. Teh, ${ }^{1}$
}

\begin{abstract}
:
This paper aims to clarify the intricacies of spatial rotation kinematics as applied to 3D stability analysis of metal framed structures with minimal mathematical abstraction. In particular, it discusses the ability of the kinematic relationships traditionally used for a spatial Euler-Bernoulli beam element, which are expressed in terms of transverse displacement derivatives, to detect the flexural-torsional instability of a cantilever and of an L-shaped frame. The distinction between transverse displacement derivatives and vectorial rotations is illustrated graphically. The paper also discusses the symmetry and asymmetry of tangent stiffness matrices derived for 3D beam elements, and the concepts of semi-tangential moments and semi-tangential rotations. Finally, the fact that the so-called vectorial rotations are independent mathematical variables are pointed out.
\end{abstract}

Keywords: buckling, finite element analysis, matrix analysis, moments, rotation, space frames

\footnotetext{
${ }^{1}$ Hon. Assoc., Dept. of Civ. Eng., Univ. of Sydney, NSW 2006, Australia
} 


\section{Introduction}

As evident from a number of journal and conference papers published in the past few years (Hsiao \& Lin 2000-2001, McGuire \& Ziemian 2000, Kim et al. 2001, Pi \& Bradford 2001, Teh 2001a), the closely related subjects of spatial rotation kinematics and flexural-torsional buckling within the general topic of 3D frame stability remain current in the structural engineering literature. The phenomenon of flexural-torsional buckling discussed by these researchers and in this paper refers to the buckling of a structural member or frame under flexure about the major axis, which is distinct from the flexural-torsional buckling of a member under axial compression due to the shear-centre eccentricity of the thin-walled open section. It is also evident that fundamental differences in opinion still exist among researchers in the field, and that reliable commercial computer programs for comprehensive 3D buckling analysis of steel frames are not widely available. Some researchers in steel frame design neglect the possibility of flexural-torsional buckling by assuming that this mode of out-ofplane instability is prevented by lateral bracing of the members of a steel frame.

Since flexural-torsional buckling is a practical reality for steel members and frames (Hancock \& Trahair 1979, Trahair 1993, McGuire \& Ziemian 2000), it is imperative that the inconsistencies and the controversies existing in the literature regarding spatial rotation kinematics as applied to flexural-torsional stability analysis be sorted out in plain English, and that the correct theories be identified. To ignore the fundamental differences between the various formulations of beam elements are unsatisfactory, especially in an era when "direct” nonlinear structural analysis and design methods are replacing the more traditional methods of steel frame design that make use of empirical formulae to account for out-of-plane buckling modes. As they are, direct methods of steel frame design rely on nonlinear finite (beam) 
element analysis programs. The tangent stiffness matrix used in the second-order elastic analysis is the same as that used in the linear buckling analysis. It is only when the correct theories for 3D frame stability analysis are identified that meaningful advancements in rational design methods of steel frames are possible.

This paper discusses three major issues in the flexural-torsional stability analysis of steel members and frames using the finite (beam) element method, namely: the energy equation for flexural-torsional buckling of a straight beam, the work-conjugacy between moments and rotations, and the concept of semi-tangential bending moments. In addition, the symmetry of element tangent stiffness matrices, the properties of the so-called vectorial rotations, and the concept of “commutative” semi-tangential rotations in geometrically nonlinear analysis are also discussed. Two examples are used to illustrate the implications of different formulations.

Due to limited space, in discussing the issues mentioned in the preceding paragraph, this paper makes references to relevant publications for the detailed formulations. However, essential equations are provided and the discussions are comprehensive, although it is assumed that the reader is reasonably familiar with the nonlinear finite element analysis method and the subject of flexural-torsional buckling.

\section{Kinematic relationships in the energy equation for flexural- torsional buckling of a straight beam}

In the literature, the following kinematic relationships of a spatial Euler-Bernoulli beam are commonly used (Argyris et al. 1979, Teh \& Clarke 1999a, McGuire et al. 2000 in section A.3)

$$
u_{x}=u-y v^{\prime}-z w^{\prime}
$$




$$
\begin{aligned}
& u_{y}=v-z \theta_{x} \\
& u_{z}=w+y \theta_{x}
\end{aligned}
$$

in which $u_{x}, u_{y}$ and $u_{z}$ are the translations of an arbitrary point $\mathrm{N}$ located at the coordinate $(y$, $z$ ) on the cross-section where the centroid $\mathrm{O}$ is subject to the translations $u, v$ and $w$ in the $x$-, $y$ - and $z$-axes of the local coordinate system defined in Fig. 1 . The variable $\theta_{x}$ represents the rotation of the cross-section about the longitudinal $x$-axis. The prime denotes differentiation with respect to the $x$-axis. Figure 2 illustrates the Euler-Bernoulli hypothesis for a 2D beam element, where the translation $u_{x}$ of the point $\mathrm{N}$ can be related to the rotation of the crosssection about the z-axis. As indicated in Fig. 2, the rotation $\theta_{z}$ about the $z$-axis is approximated by the transverse displacement derivative $v^{\prime}$.

The kinematic relationship (1) neglects the longitudinal displacement due to torsional warping, which is significant for wide-flange sections. However, this aspect is irrelevant to the present discussion and all the discussions in subsequent sections of this paper.

Pi \& Bradford (2001) point out that the use of the kinematic relationships (1)-(3) is equivalent to the use of a so-called "small rotation matrix"

$$
\mathbf{R}_{1}=\left[\begin{array}{ccc}
1 & -\theta_{x} & v^{\prime} \\
\theta_{x} & 1 & w^{\prime} \\
-v^{\prime} & -w^{\prime} & 1
\end{array}\right]
$$

as the kinematic relationships (1)-(3) can be expressed in vector/matrix format using $\mathbf{R}_{1}$. 
Pi \& Bradford (2001) state that the use of the kinematic relationships (1)-(3) in formulating the energy equation leads to a beam element that is unable to predict the correct flexuraltorsional buckling load of a beam subjected to non-uniform bending moments (and therefore, subjected to transverse shear forces). Pi \& Bradford (2001) also suggest that the researchers who use such kinematic relationships, and hence implicitly use the "small rotation matrix" $\mathbf{R}_{1}$, have apparently noted the problem and subsequently modified their finite element by adding a term which is the work done by the transverse shear forces to their energy equations. Pi \& Bradford (2001) argue that this term is "added more by engineering judgment rather than by a rigorous mechanical (sic) derivation”.

There are two points worth noting regarding the forementioned points made by Pi \& Bradford (2001). Firstly, the researchers cited by Pi \& Bradford (2001) do not appear to have stated or implied that the use of the kinematic relationships (1)-(3) leads to a flawed energy equation for flexural-torsional buckling (of an isolated straight beam). Secondly, at least one of them (Rajasekaran 1977) shows that the virtual work term due to transverse shear forces result from the integration of the transverse shear stresses over the cross-section area. (It should be noted that this integration is independent of the Euler-Bernoulli hypothesis, which is a convenient assumption for the kinematic relationships. This aspect points to an advantage of the energy methods of formulating equilibrium equations, but this topic is outside the scope of this paper.)

The fact that the presence or absence of the (virtual) work terms due to transverse shear forces has nothing to do with the use of the "small rotation matrix" $\mathbf{R}_{1}$ is also evident from the detailed Updated Lagrangian formulation of McGuire et al. (2000). McGuire et al. (2000) employ the kinematic relationships (1)-(3) in section A.3 of their textbook, and show that the 
virtual work terms due to transverse shear forces (eg. $\left.\int_{L} F_{z} \delta\left(\theta_{x} v^{\prime}\right) d x\right)$ are obtained from the integration of the transverse shear stresses over the cross-section area (eg. $\int_{A} \tau_{x z} d A=F_{z}$ ), which are substituted into the internal virtual work functional. Importantly, section A.3, which contains this derivation, precedes section A.4 that employs a "finite rotation matrix" expressed to the second order.

Pi \& Bradford (2001) present a flexural-torsional buckling analysis example of a cantilever subjected to a transverse shear force at the tip. This example is intended to demonstrate that the use of the "small rotation matrix" $\mathbf{R}_{1}$, and therefore the kinematic relationships (1)-(3), leads to failure of the derived beam element to predict the correct flexural-torsional buckling load of the cantilever. Pi \& Bradford (2001) state that the use of four such elements grossly underestimates the linear flexural-torsional buckling load of the cantilever.

However, the issue concerning the effect of finite rotation kinematics on flexural-torsional buckling analysis using spatial beam elements, pioneered by Argyris et al. (1978), is only relevant to framed structures in which the members are connected non-collinearly. When the members (or elements) are connected collinearly, as in the case of a cantilever model, all the additional terms in the element tangent stiffness matrices "due to the use of the second-order rotation matrix" vanish, as pointed out later in Section 5. It can be shown that the beam element derived by McGuire et al. (2000, section A3) using the kinematic relationships (1)(3), and thus the "small rotation matrix" $\mathbf{R}_{1}$ shown in Equation (4), is able to accurately predict the flexural-torsional buckling load of a cantilever subjected to a transverse shear force at the tip. 
For the cantilever in example 9.11 of the textbook by McGuire et al. (2000), shown in Fig. 3, the use of four such elements results in an error of $1 \%$ only. It should be reiterated that all the additional moment terms due to the second-order rotation matrix described by McGuire et al. (2000) in section A.4 of their textbook vanish totally in modelling the cantilever since only collinearly connected beam elements are involved.

\section{Work-conjugacy between bending moments and rotation parameters}

In the literature, the bending moment about the $z$-axis $M_{\mathrm{z}}$ is widely assumed to be workconjugate with the first transverse displacement derivative $v^{\prime}$. This assumption is fine for 2D frame analysis, but is unjustified in out-of-plane buckling analysis as will be seen later. The fundamental issue of work-conjugacy between bending moments and rotation parameters has, for one reason or another, been neglected by most researchers in stability analysis of space frames. This neglect has contributed to the introduction of the so-called semi-tangential bending moments (Argyris et al. 1978), discussed in the next section, and is the source of much confusion in the 3D nonlinear frame analysis literature.

Crisfield (1997, page 249) cites from Ziegler (1968) that moments about fixed axes are nonconservative, and argues that such moments will lead to an asymmetric tangent stiffness matrix. Crisfield (1997) then states that “it is not difficult to apply local 'follower moments' at the nodes. For example, if such moments were applied in relation to the local element axes at node 1, one could consider the external work to be given by $V_{\mathrm{e}}=M_{\mathrm{e} 1} \delta \theta_{l 1}+M_{\mathrm{e} 2} \delta \theta_{l 2}+M_{\mathrm{e} 3} \delta \theta_{l 3} \ldots$ ” However, follower moments have also been shown to be non-conservative (Argyris et al. 1978), just like the moments about fixed axes. Crisfield 
(1997, page 251) alludes to the 'anomalies' of gravity loading with the Co-rotational formulation.

On the other hand, Teh \& Clarke (1997) and Trahair \& Teh (2001) point out that, contrary to the popular belief among computational mechanics researchers, a conservative moment needs not be made up of forces fixed in direction. Teh \& Clarke (1997) propose a new definition of conservative moment that, within a certain range of rotations, behaves like a follower (tangential) moment. The work expression for such moments is (Teh \& Clarke 1997)

$$
W=M_{x} \theta_{x}+M_{y} \theta_{y}+M_{z} \theta_{z}
$$

in which $\theta_{x}, \theta_{y}$ and $\theta_{z}$ are the Cartesian components of the resultant rotation $\boldsymbol{\theta}$, as defined in Fig. 4.

In the present work, the Cartesian components $\theta_{x}, \theta_{y}$ and $\theta_{z}$ of the resultant rotation $\boldsymbol{\theta}$ are called the "vectorial rotations". However, it should be noted that the rotation $\theta$ itself is not a vector (Oran 1973), and has been termed the rotational pseudo-vector (Crisfield 1990).

The work expression (5) has an important implication in the formulation of a spatial beam element using an energy principle. For a virtual work equation to be meaningful, the (virtual) nodal displacement vector in the external virtual work expression $V_{\mathrm{e}}$ must contain the vectorial rotations as the nodal rotation variables

$$
\begin{aligned}
& \text { Internal virtual work } V_{\mathrm{i}}=\text { External virtual work } V_{\mathrm{e}} \\
& V_{\mathrm{e}}=\mathbf{f} \delta \mathbf{d} \\
& \mathbf{f}=\left\{F_{x 1} F_{y 1} F_{z 1} M_{x 1} M_{y 1} M_{z 1} F_{x 2} F_{y 2} F_{z 2} M_{x 2} M_{y 2} M_{z 2}\right\}
\end{aligned}
$$




$$
\mathbf{d}=\left\{\begin{array}{llllllllllll}
u_{1} & v_{1} & w_{1} & \theta_{x 1} & \theta_{y 1} & \theta_{z 1} & u_{2} & v_{2} & w_{2} & \theta_{x 2} & \theta_{y 2} & \theta_{z 2}
\end{array}\right\}^{\mathrm{T}}
$$

The variables in the nodal force vector $\mathbf{f}$ and the nodal displacement vector $\mathbf{d}$ are defined in Fig. 5.

Donald \& Kleeman (1977) presented the following second-order relationships

$$
\begin{aligned}
& v^{\prime}=\theta_{z}+\frac{1}{2} \theta_{x} \theta_{y} \\
& w^{\prime}=-\theta_{y}+\frac{1}{2} \theta_{x} \theta_{z}
\end{aligned}
$$

The second-order relationships (10)-(11) are illustrated graphically in Fig. 6, which depicts the final position of a rigid rod of length $L$ initially aligned with the $x$-axis following a rotation $\boldsymbol{\theta}$ centred at one end.

The second-order relationships (10)-(11) indicate that, in the application of the virtual work principle to the derivation of a spatial beam element, the virtual nodal transverse displacement derivatives $\delta v_{1}^{\prime}$ and $-\delta w_{1}^{\prime}$ (over which the virtual transverse displacement derivatives along the beam element are interpolated) in the internal virtual work expression $V_{\mathrm{i}}$ do not eliminate the virtual nodal vectorial rotations $\delta \theta_{z 1}$ and $\delta \theta_{y 1}$ in the external virtual work expression $V_{\mathrm{e}}$, respectively. This is the point often missed in the literature as discussed in the following section. 


\section{Additional terms due to "finite rotations"}

Argyris et al. (1978) state that if the internal bending moment of a spatial beam is considered to be "quasi-tangential" while the St. Venant torque is "semi-tangential", then a joint that is initially in equilibrium under a bending moment $M$ on one side and a torque $T$ on the other side will lose equilibrium under the same forces when it undergoes a finite rotation. This point is illustrated by McGuire et al. (2000) using Fig. 7. McGuire et al. (2000) state that under a rigid body rotation $\theta_{\mathrm{x}}$, a moment increment of $-M \theta_{x}$ about the $y$-axis is generated by the quasi-tangential bending moment $M$, while only half that increment is generated in the opposite direction by the semi-tangential torque $T$. They contend that this problem is due to the use of the conventional engineering theories of flexure and torsion.

In order to resolve the perceived problem illustrated in Fig. 7, and to address the fact that their original tangent stiffness matrix cannot correctly predict the flexural-torsional buckling load of an L-shaped frame, Argyris et al. (1978) introduced the concept of semi-tangential bending moments. Under a semi-tangential bending moment and a semi-tangential torque, equal and opposite moment increments of $1 / 2 M \theta_{x}$ are generated. This concept cannot be justified as it means that the internal bending moment of a beam, which is a resultant of the normal stresses acting on the cross-section, is represented in the manner depicted in Fig. 8.

On the other hand, McGuire et al. (2000, section A4) employ a second-order rotation matrix

$$
\mathbf{R}_{2}=\left[\begin{array}{ccc}
1-1 / 2\left(\theta_{y}{ }^{2}+\theta_{z}{ }^{2}\right) & -\theta_{z}+1 / 2 \theta_{x} \theta_{y} & \theta_{y}+1 / 2 \theta_{x} \theta_{z} \\
\theta_{z}+1 / 2 \theta_{x} \theta_{y} & 1-1 / 2\left(\theta_{x}{ }^{2}+\theta_{z}{ }^{2}\right) & -\theta_{x}+1 / 2 \theta_{y} \theta_{z} \\
-\theta_{y}+1 / 2 \theta_{x} \theta_{z} & \theta_{x}+1 / 2 \theta_{y} \theta_{z} & 1-1 / 2\left(\theta_{x}{ }^{2}+\theta_{y}{ }^{2}\right)
\end{array}\right]
$$


to express the kinematic relationships of their beam element

$$
\left\{\begin{array}{l}
u_{x} \\
u_{y} \\
u_{z}
\end{array}\right\}=\left\{\begin{array}{l}
u \\
v \\
w
\end{array}\right\}+\left(\mathbf{R}_{2}-\mathbf{I}\right)\left\{\begin{array}{l}
0 \\
y \\
z
\end{array}\right\}
$$

in which $\mathbf{I}$ is an identity matrix. It may be noted that the elements of the second-order rotation matrix $\mathbf{R}_{2}$ are not positioned in the same way as those of the "small rotation matrix" $\mathbf{R}_{1}$ shown in Equation (4). This is so as McGuire et al. (2000) and Pi \& Bradford (2001) use slightly different matrix formats in expressing the kinematic relationships.

The use of the second-order rotation matrix $\mathbf{R}_{2}$ leads to additional moment terms in the geometric stiffness matrix (Chen 1994, McGuire et al. 2000)

$$
\mathbf{k}_{\mathrm{G}}=\frac{1}{2}\left[\begin{array}{cccccc}
\boldsymbol{\theta}_{x 1} & \boldsymbol{\theta}_{y 1} & \boldsymbol{\theta}_{z 1} & \boldsymbol{\theta}_{x 2} & \boldsymbol{\theta}_{y 2} & \boldsymbol{\theta}_{z 2} \\
0 & -M_{z 1} & M_{y 1} & 0 & 0 & 0 \\
& 0 & 0 & 0 & 0 & 0 \\
& & 0 & 0 & 0 & 0 \\
& \text { Sym. } & & 0 & -M_{z 2} & M_{y 2} \\
& & & & 0 & 0 \\
& & & & & 0
\end{array}\right]
$$

The nodal moment terms in the geometric stiffness matrix (14) originate from the internal bending stress integrals of the internal virtual work expression.

Through flexural-torsional buckling analyses of the L-shaped frame shown in Fig. 9, Chen (1994) and McGuire et al. (2000) found that the neglect of the terms shown in Equation (14) resulted in the predicted elastic critical load being approximately half of the correct value. The correct value was verified using shell elements, as shown in Table 1. 
There are a number of points worth noting regarding the Updated Lagrangian formulation of Chen (1994) and McGuire et al. (2000). Firstly, the additional matrix shown in Equation (14) coincides with the semi-tangential adjustment matrix of Argyris et al. (1979). Secondly, the derived beam element does not pass spatial rigid body motion tests (Teh \& Clarke 1996a). In other words, under a rigid body rotation of the element without any straining, the element tangent stiffness matrix (often used as the incremental stiffness matrix) does not yield zero force increments. Thirdly, the formulation does not explain why the element tangent stiffness matrices derived in the Co-rotational framework are asymmetric, even though the same rotation variables are used. This issue is discussed further in the next section.

Some authors such as Elias (1986) and Conci \& Gattass (1990) are aware of the second-order relationships (10)-(11). They also recognise that transverse displacement derivatives are not continuous between connected members of a space frame. However, they implicitly assume the nodal bending moments of a spatial beam to be work-conjugate with the corresponding nodal transverse displacement derivatives rather than the corresponding nodal vectorial rotations. Accordingly, they attempt to account for the second-order relationships (10)-(11) in the external virtual work expression $V_{\mathrm{e}}$ of their Updated Lagrangian formulations, instead of the internal virtual work expression $V_{\mathrm{i}}$. The resulting additional stiffness matrix is therefore opposite to that shown in Equation (14), as can be seen from equation (51) given by Conci \& Gattass (1990).

It should also be noted that the use of the "second-order rotation matrix" described by Pi \& Bradford (2001), which is expressed in terms of transverse displacement derivatives rather than vectorial rotations, does not necessarily result in the additional stiffness matrix (14). Pi \& Bradford (2001) implicitly assume the bending moments to be work-conjugate with the 
corresponding transverse displacement derivatives, and do not convert the transverse displacement derivatives into vectorial rotations.

As far as flexural-torsional buckling analysis of non-collinearly connected members under transferred bending moment is concerned, Equation (14) is sufficient. Its coincidence with the semi-tangential adjustment matrix of Argyris et al. (1979) is discussed in the following section.

\section{Other intricate issues associated with spatial rotations}

By enforcing the semi-tangential behavior of nodal bending moments into the geometric stiffness matrix, Argyris et al. (1979) also obtain the additional moment terms shown in Equation (14). [It is worth noting that, contrary to statements by other authors, Argyris et al. (1979) employ transverse displacement derivatives instead of semi-tangential rotations in their 1979 publication.] On the other hand, these terms are derived directly by Chen (1994) and McGuire et al. (2000) by assuming the bending moments to be work-conjugate with the corresponding vectorial rotations, as expressed in Equation (5). As mentioned previously, the work expression (5) is valid for moments that behave in a follower (tangential) manner over the resultant rotation. However, Teh \& Clarke (1997) have stated that the work expression (5) is also valid for a semi-tangential moment defined by Ziegler (1968), despite its unique rotational behavior.

Under conservative loadings, a tangent stiffness matrix $\mathbf{k}_{\mathrm{T}}$ (which includes the geometric stiffness matrix) derived directly from an energy principle is invariably symmetric (Oran 1973, Teh \& Clarke 1999b), since 


$$
k_{\mathrm{T} i j}=\frac{\partial^{2} U}{\partial d_{i} \partial d_{j}}=\frac{\partial^{2} U}{\partial d_{j} \partial d_{i}}=k_{\mathrm{T} j i}
$$

provided the strain energy $U$ of the conservative structure is expressed as a continuous function of the displacement variables. Whether the displacement variables $d_{\mathrm{i}}$ and $d_{\mathrm{j}}$ are commutative to each other, or whether they are work-conjugate with the corresponding force variables, is irrelevant to Equation (15). The equation is merely a mathematical fact, and says nothing about the correctness of the derived tangent stiffness matrix. In the literature, the order of differentiation of the strain energy function $U$ has often been confused with the order of displacements applied to the element during the geometry update, which led to the concept of commutative (additive) semi-tangential rotations (Argyris et al. 1978).

In an Updated Lagrangian formulation where the loads are "independent" of the displacements, the element tangent stiffness matrix is indeed derived solely as the second partial derivatives of the strain energy function, as expressed by Equation (15). In the elastic realm, the principle of stationary potential energy is equivalent to the principle of virtual work. As a result, the Updated Lagrangian formulation of Chen (1994) and McGuire et al. (2000), which implicitly assumes the bending moments to be work-conjugate with the corresponding vectorial rotations, yields a symmetric tangent stiffness matrix (more precisely, geometric stiffness matrix) which implies the semi-tangential behavior of the nodal bending moments and torque.

The semi-tangential tangent stiffness matrix does not give zero force increments under spatial rigid body rotation of the element. The concept of semi-tangential bending moments, which was introduced by Argyris et al. (1978) to resolve the perceived loss of joint equilibrium illustrated in Fig. 7, was only viable owing to the mistaken belief that a conservative moment 
must be invariably made up of forces fixed in direction. If this restriction is removed, then there should be no loss of joint equilibrium as the plane of the torque $T$ in Fig. 7 rotates fully with the cross-section (Teh \& Clarke 1997, Trahair \& Teh 2001).

Unlike the Updated Lagrangian formulation which directly accounts for the rigid body rotations of the element via the nodal translations, the Co-rotational formulation excludes the rigid body modes during the derivation of the element stiffness matrices using an energy principle. More detailed descriptions of the Updated Lagrangian and the Co-rotational formulations can be found in Teh \& Clarke (1998) and Teh (2001b). In order to account for the change in orientation of the nodal forces during rigid body rotations, a stability matrix (often called the external geometric stiffness matrix) is added to the element tangent stiffness matrix. By enforcing the tangential behavior of nodal moments, Teh \& Clarke (1998) obtained an asymmetric stability matrix, resulting in an asymmetric element tangent stiffness matrix.

Other researchers who use the Co-rotational formulation have also derived asymmetric tangent stiffness matrices (Crisfield 1990, Spillers et al. 1993, Hsiao \& Lin 2000). They employ the work expression (5), which involves work-conjugate moment and rotation variables. It is also noteworthy that Cardona \& Geradin (1988) obtain symmetric stiffness matrices using the Total and the Updated Lagrangian formulations, but an asymmetric stiffness matrix using the alternative formulation. These results indicate that the symmetry or asymmetry of a tangent stiffness matrix is not necessarily caused by the rotation variables used to derive the finite element, or the work-conjugacy between the adopted moment and rotation definitions. 
If one enforces the tangential behavior of nodal moments into the formulation of Argyris et al. (1979), which implicitly assumes the bending moments to be work-conjugate with the corresponding transverse displacement derivatives, then the following asymmetric correction stiffness matrix will be obtained instead of the symmetric one shown in Equation (14)

$$
\mathbf{k}_{\mathrm{G}}=\frac{1}{2}\left[\begin{array}{ccc}
\theta_{x i} & \theta_{y i} & \theta_{z i} \\
0 & 0 & 0 \\
-2 M_{z i} & 0 & M_{x i} \\
2 M_{y i} & -M_{x i} & 0
\end{array}\right] ; i=1,2
$$

Teh \& Clarke (1996a) show that the Updated Lagrangian cubic beam element that includes the correction stiffness matrix (16) passes the spatial rigid body motion test.

However, Teh \& Clarke (1999b) have demonstrated through a heuristic example that in the direct stiffness method of matrix structural analysis, the asymmetric part of the correction stiffness matrix (16) vanishes at equilibrium when the element tangent stiffness matrix is assembled to the structure tangent stiffness matrix. This demonstration is in agreement with the more rigorous theoretical treatment by Simo \& Vu-Quoc (1986) and Nour-Omid \& Rankin (1991). The symmetric part of the correction stiffness matrix (16) coincides with the additional stiffness matrix (14), and hence the semi-tangential adjustment matrix, for

$$
{ }^{\text {symm }} \mathbf{k}_{\mathrm{G}}=\frac{\mathbf{k}_{\mathrm{G}}+\mathbf{k}_{\mathrm{G}}{ }^{\mathrm{T}}}{2}=\frac{1}{2}\left[\begin{array}{ccc}
0 & \theta_{y i} & \theta_{z i} \\
& M_{y i} \\
0 & 0 \\
\text { symmetric } & 0
\end{array}\right] ; i=1,2
$$


When the modified Newton-Raphson iteration method is used, in which case the structure tangent stiffness matrix is determined at the start of each new increment only following the convergence of the last increment, the correction stiffness matrix (16) reduces to the additional stiffness matrix (14). A symmetrised tangent stiffness matrix can therefore be employed in conjunction with the modified Newton-Raphson iteration method (Teh \& Clarke 1999b), resulting in much efficiency. This is also true for linear buckling analysis as the computed element forces will always be in equilibrium with each other. In fact, even when the full Newton-Raphson iteration method is employed in a nonlinear analysis, the use of a symmetrised tangent stiffness matrix only marginally impairs the convergence rate.

It is also evident that, for two collinearly connected elements, the correction stiffness matrix (16) vanishes altogether at nodal equilibrium as the moment terms cancel each other totally. Therefore, with respect to linear buckling analysis, the issue of second-order rotation terms does not arise for collinearly connected elements, including those modelling a cantilever. This fact also means that a computer program that can predict the flexural-torsional buckling load of a beam structure correctly cannot necessarily predict same of a framed structure.

\section{Are vectorial rotations independent variables?}

As depicted in Fig. 4, the vectorial rotations $\theta_{x}, \theta_{y}$ and $\theta_{z}$ are the Cartesian components of a resultant rotation $\boldsymbol{\theta}$. However, it is well-known that "physical” rotations about orthogonal axes are not commutative to each other, that is, they cannot be summed up as vectors. Therefore, the vectorial rotations $\theta_{x}, \theta_{y}$ and $\theta_{z}$ are not three actual rotations about the respective axes, but are convenient mathematical parameters used in handling the kinematics of spatial rotations. This fact has been used by some researchers (Kim et al. 2001) to argue that the three 
orthogonal vectorial rotations were not independent of each other, and therefore could not be used in formulating a spatial beam element.

However, if the vectorial rotations $\theta_{x}, \theta_{y}$ and $\theta_{z}$ were not independent variables as 'they are merely the Cartesian components of a single resultant rotation of the magnitude $\theta=\sqrt{\theta_{x}{ }^{2}+\theta_{y}{ }^{2}+\theta_{z}{ }^{2}}$, then it could also be argued that the translations $u_{x}, u_{y}$ and $u_{z}$ were not independent variables as they are the Cartesian components of a single resultant translation of the magnitude $u=\sqrt{u_{x}{ }^{2}+u_{y}{ }^{2}+u_{z}{ }^{2}}$. The truth is, of course, the vectorial rotations $\theta_{x}, \theta_{y}$ and $\theta_{z}$ are independent mathematical variables, just like the translation components $u_{x}, u_{y}$ and $u_{z}$.

In a nonlinear frame analysis using load incrementation and equilibrium iteration, each step is linearised and a node of the frame model undergoes only one resultant rotation $\theta$ in a step. The fact that successive incremental rotations $\theta$ are not commutative to each other can be handled using the joint orientation approach (Oran 1973) or its variants (Teh \& Clarke 1996b). There is no need to employ the concept of semi-tangential rotations as there is no reason why successive joint rotations of a structure should be able to take place in any order.

\section{Concluding remarks}

The kinematic relationships traditionally employed for spatial Euler-Bernoulli beam elements do not necessarily result in a flawed energy equation for the flexural-torsional buckling analysis of straight beams subjected to non-uniform bending moments (or transverse shear forces). At least in the Updated Lagrangian formulation, the virtual work terms due to 
transverse shear forces, which are said to be crucial, have been derived consistently by some researchers when such kinematic relationships are employed.

For the derivation of a spatial beam element that can be used for flexural-torsional buckling analysis of framed structures in which the members are connected non-collinearly, the second-order rotation matrix expressed in terms of vectorial rotations should be used in expressing the kinematic relationships. The key point is in recognising that the bending moments are work-conjugate with vectorial rotations, and not with transverse displacement derivatives.

The concept of semi-tangential bending moments was only viable due to the mistaken belief that a conservative moment must invariably be made up of forces fixed in direction, and to the assumption that bending moments are work conjugate with transverse displacement derivatives in the 3D realm. Nevertheless, a beam element that is derived using this concept, or of which nodal bending moments exhibit semi-tangential behavior, is able to accurately predict the flexural-torsional buckling load of a framed structure in which the members are connected non-collinearly. The work expressions for a semi-tangential moment and for a tangential moment, the latter representing the true behaviour of nodal moments under spatial rotations, are the same.

Only the tangent stiffness matrix that exhibits the tangential behavior of nodal moments passes the spatial rigid body motion test. Such a stiffness matrix is asymmetric due to some nodal moment terms. However, at nodal equilibrium, the asymmetric tangent stiffness matrix becomes symmetrised. A symmetrised tangent stiffness matrix can therefore be used to predict the flexural-torsional instability of framed structures. 
For collinearly connected elements, such as those modelling a cantilever, the additional moment terms vanish totally at equilibrium, not just becoming symmetric. Therefore, the issue of finite rotations in space does not arise in 3D linear buckling analysis of a straight beam structure. A computer program that can predict the flexural-torsional buckling load of a straight beam structure may not be able to perform same of a framed structure in which the members are connected non-collinearly. It is important to benchmark a 3D nonlinear frame analysis program against the L-shaped frame example, without introducing any geometric imperfections or load perturbations to initiate the lateral buckling.

The concept of "commutative" semi-tangential rotations arises from the confusion between the order of differentiation of the strain energy function and the order of rotations applied to the element during the geometry update. A tangent stiffness matrix of a conservative system derived directly from an energy principle will always be symmetric provided the strain energy is expressed as a continuous function of the displacement variables, irrespective of its correctness. The symmetry and asymmetry of many tangent stiffness matrices presented in the literature have nothing to do with the work-conjugacy between the adopted nodal moment and rotation definitions or with the rotation parameters used in the element formulation, but are rather due to the frameworks in which the formulations are cast (whether Lagrangian or Corotational), and to the rotational behavior of nodal moments assumed in deriving the corresponding geometric stiffness matrices.

The vectorial rotation variables, which are the Cartesian components of a single resultant rotation, are independent variables suitable for deriving a spatial beam element to be used in 3D stability analysis of steel frames. 


\section{References}

Argyris, J.H., Dunne, P. C. and Scharpf, D. W. (1978) “On large displacement - small strain analysis of structures with rotational degrees of freedom.” Comp. Meth. Appl. Mech. Engrg., 14, 401-451; 15, 99-135.

Argyris, J.H., Hilpert, O., Malejannakis, G.A., and Scharpf, D.W. (1979) “On the geometrical stiffnesses of a beam in space - a consistent V.W. approach.” Comp. Meth. Appl. Mech. Engrg., 20, 105-131.

Cardona, A., and Geradin, M. (1988) “A beam finite element non-linear theory with finite rotations.” Int. J. Numer. Meth. Engrg., 26, 2403-2438.

Chen, C. S. (1994) “Geometric nonlinear analysis of three-dimensional structures,” M.S thesis, Cornell University, Ithaca, NY.

Conci, A., and Gattass, M. (1990) "Natural approach for geometric nonlinear analysis of thinwalled frames.” Int. J. Numer. Meth. Engrg., 30, 207-231.

Crisfield, M. A. (1990) “A consistent Co-rotational formulation for non-linear, threedimensional beam elements.” Comp. Meth. Appl. Mech. Engrg., 81, 131-150.

Crisfield, M. A. (1997). Non-linear Finite Element Analysis of Solids and Structures, Vol. 2, John Wiley \& Sons, Chichester, England.

Donald, G. S. and Kleeman, P. W. (1971) “A stiffness matrix for the large deflection analysis of space structures.” Proc., Third Australasian conference on structures and materials, Auckland, August 1971.

Elias, Z.M. (1986). Theory and methods of structural analysis, John Wiley, NY. 
Hancock, G. J., and Trahair, N. S. (1979) "Finite element analysis of the lateral buckling of continuously restrained beam-columns,” Civ. Engrg. Trans., IEAust., CE20(2), 120-127.

Hsiao, K. M., and Lin, W. Y. (2000) “A Co-rotational formulation for thin-walled beams with mono-symmetric open sections.” Comp. Meth. Appl. Mech. Engrg., 190, 1163-1185.

Hsiao, K. M., and Lin, W. Y. (2001) “A Co-rotational formulation for geometric nonlinear analysis of doubly symmetric thin-walled beams.” Comp. Meth. Appl. Mech. Engrg., 190, 6023-6052.

Kim, M. Y., Chang, S. P., and Park, H. G. (2001) "Spatial postbuckling analysis of nonsymmetric thin-walled frames. I: Theoretical considerations based on semitangential property.” J. Engrg. Mech., ASCE, 127 (8), 769-778.

McGuire, W., and Ziemian, R. (2000) “Steel frame stability: Out-of-plane effects.” Proc., First Int. Conf. Structural Stability and Dynamics, 7-9 December 2000, Taipei, Taiwan, Y. B. Yang, L. J. Leu, and S. H. Hsieh, eds, 5-20.

McGuire, W., Gallagher, R.H., and Ziemian, R. (2000) Matrix Structural Analysis, John Wiley and Sons, New York.

Nour-Omid, B., and Rankin, C.C. (1991) "Finite rotation analysis and consistent linearisation using projectors.” Comp. Meth. Appl. Mech. Engrg., 93, 353-384.

Oran, C. (1973) “Tangent stiffness in space frames.” J. Struct. Div., ASCE, 99, 987-1001.

Pi, Y. L., and Bradford, M. A. (2001) "Effects of approximations in analyses of beams of open thin-walled cross-section- Part I: Flexural-torsional instability,” Int. J. Numer. Meth. Engrg., 51, 757-772. 
Rajasekaran, S. (1977) “Finite element method for plastic beam-columns.” Theory of BeamColumns Volume 2: Space Behaviour and Design, W. F. Chen and T. Atsuta, eds., McGraw-Hill, New York, 539-606.

Simo, J. C., and Vu-Quoc, L. (1986) “A three dimensional finite strain rod model, Part II: Computational aspects.” Comp. Meth. Appl. Mech. Engrg., 58, 79-115.

Spillers, W. R., Saadeghvaziri, A., and Luke, A. (1993) “An example of three-dimensional frame buckling.” Comp. Struct., 47, 483-486.

Teh, L. H. (2001a) "Second-order terms and benchmarking in 3D frame analysis.” Proc., $6^{\text {th }}$ Pacific Structural Steel Conf., 15-17 Oct 2001, Beijing, China, W. Liu and B. Wang, eds., Seismological Press, Beijing, 190-195.

Teh, L. H. (2001b) “Cubic beam elements in practical analysis and design of steel frames.” Engrg. Struct., 23 (10), 1243-1255.

Teh, L. H., and Clarke, M. J. (1996a) “An Updated Lagrangian formulation for second-order elastic analysis of space frames using beam elements.” Research Report No. R727, School of Civil and Mining Engineering, University of Sydney, Australia.

Teh, L. H., and Clarke, M. J. (1996b) “The implications of linearisations, approximations and computation algorithms in the second-order analysis of frames.” Research Report No. R733, School of Civil and Mining Engineering, University of Sydney, Australia.

Teh, L. H., and Clarke, M. J. (1997) "New definition of conservative internal moments in space frames.” J. Engrg. Mech., ASCE, 123 (2), 97-106.

Teh, L. H., and Clarke, M. J. (1998) “Co-rotational and Lagrangian formulations of elastic three-dimensional beam finite elements,” J. Construct. Steel Res., 48, 123-144. 
Teh, L. H., and Clarke, M. J. (1999a) "Plastic-zone analysis of 3D steel frames using beam elements.” J. Struct. Engrg., ASCE, 125 (11), 1328-1337.

Teh, L. H., and Clarke, M. J. (1999b) "Symmetry of tangent stiffness matrices of 3D elastic frame.” J. Engrg. Mech., ASCE, 125 (2), 248-251.

Trahair, N. S. (1993) Flexural-Torsional Buckling of Structures, E\&FN Spon, London.

Trahair, N. S., and Teh, L. H. (2001) “Second-order moments in torsion members.” Engrg. Struct., 23 (6), 631-642.

Ziegler, H. (1968). Principles of structural stability, Blaisdell, London. 
Table 1 Flexural-torsional buckling load of L-shaped frame

\begin{tabular}{|l|c|c|c|c|}
\hline & Type of Element & Number & Eqn (14) & $\boldsymbol{P}_{\text {cr }}$ \\
\hline Argyris et al (1979) & Triangular shell & 86 & N/A & 1.163 \\
\hline Nour-Omid \& Rankin (1991) & Quadrilateral shell & 64 & N/A & 1.130 \\
\hline McGuire et al (2000, section A3) & Cubic beam & 8 & No & 0.551 \\
\hline McGuire et al (2000, section A4) & Cubic beam & 8 & Yes & 1.093 \\
\hline
\end{tabular}




\section{List of figures}

Figure 1 Local coordinate system of beam element

Figure 2 Euler-Bernoulli assumption of sections remaining plane and normal to the centroidal line

Figure 3 Flexural-torsional buckling analysis of cantilever (McGuire et al. 2000)

Figure 4 Vectorial rotation variables

Figure 5 Nodal forces and nodal displacements of spatial beam element

Figure 6 Graphical illustration of Equations (10) and (11)

Figure 7 Loss of joint equilibrium under rotation (McGuire et al. 2000)

Figure 8 Semi-tangential bending moment

Figure 9 Clamped angle frame under a transverse tip load (Argyris et al 1979) 


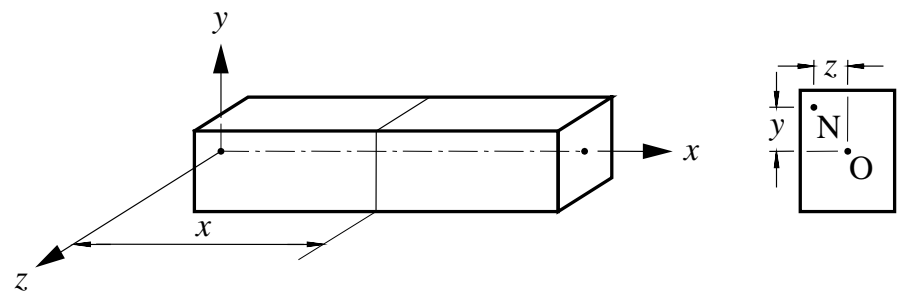

Figure 1 Local coordinate system of beam element 


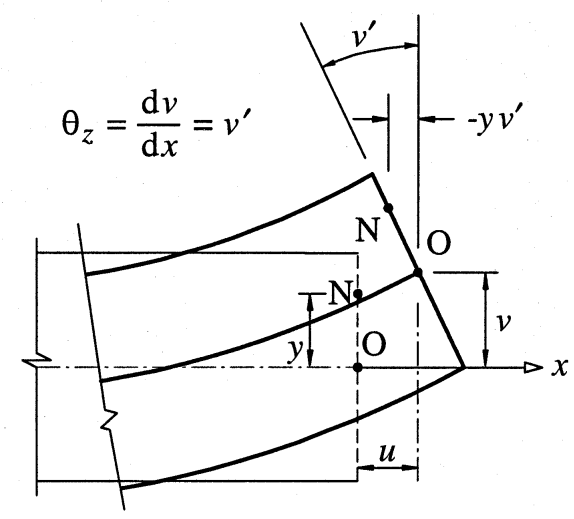

Figure 2 Euler-Bernoulli assumption of sections remaining plane and normal to the centroidal line 


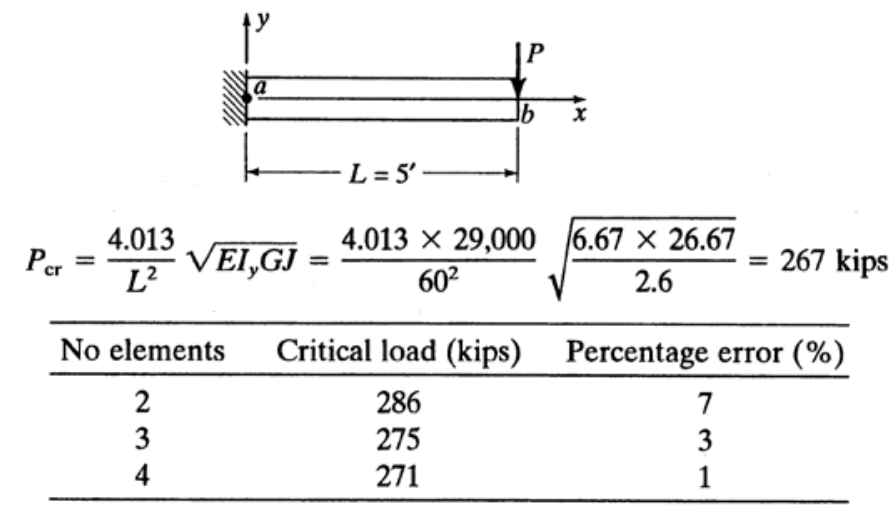

Figure 3 Flexural-torsional buckling analysis of cantilever (McGuire et al. 2000) 


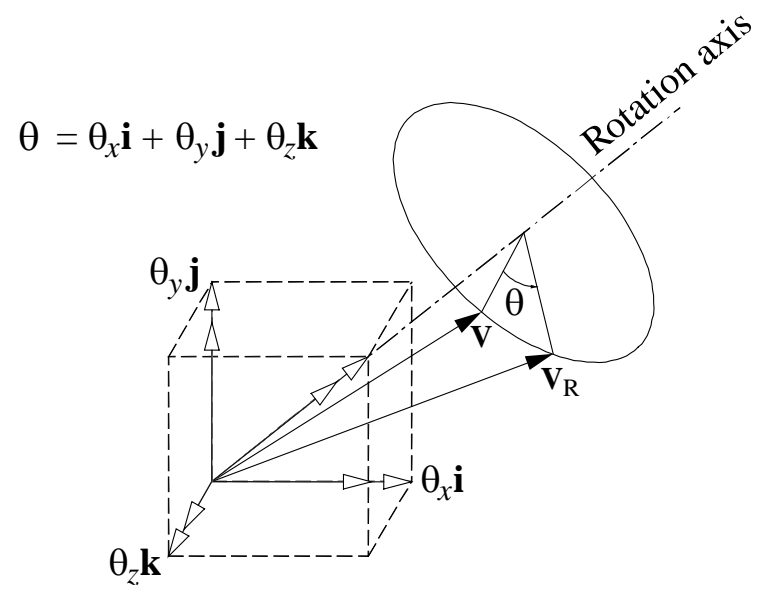

Figure 4 Vectorial rotation variables 


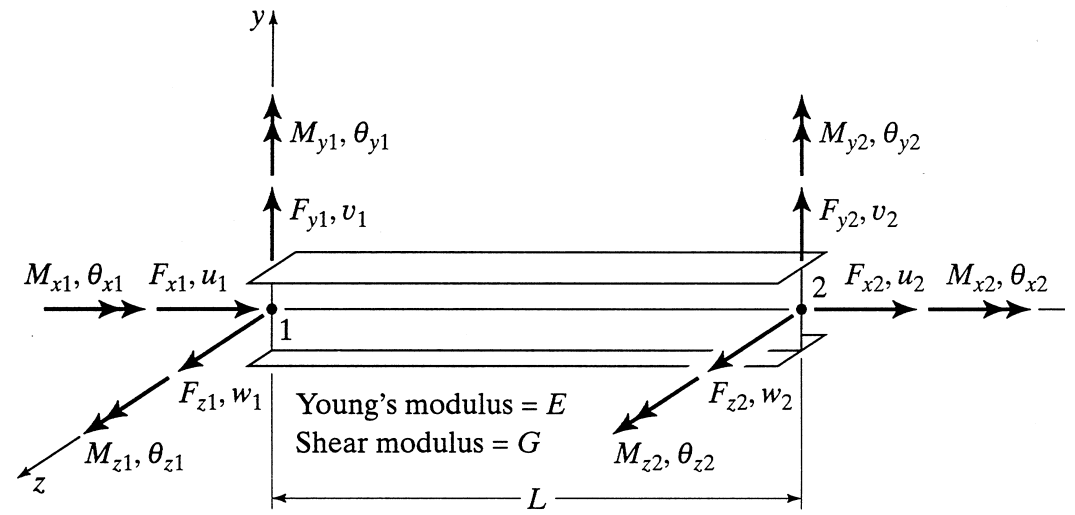

Figure 5 Nodal forces and nodal displacements of spatial beam element 


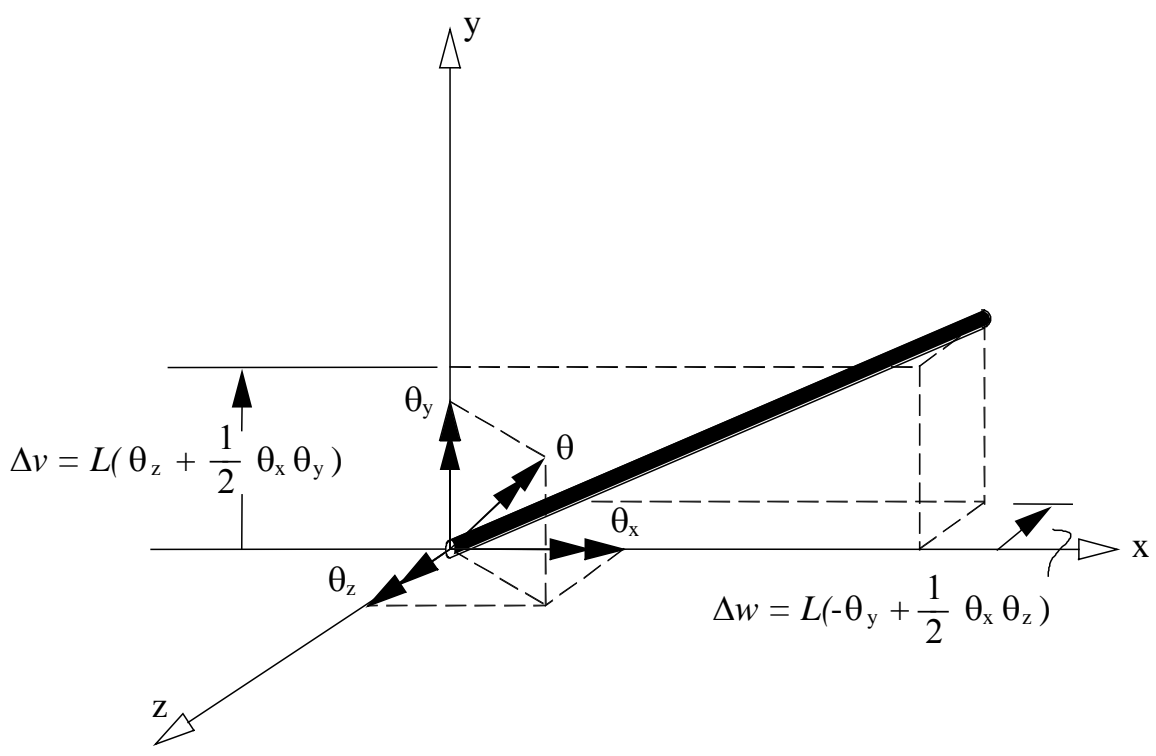

Figure 6 Graphical illustration of Equations (10) and (11) 


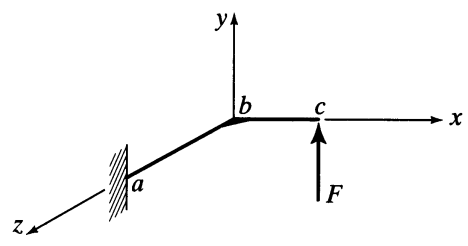

(a)

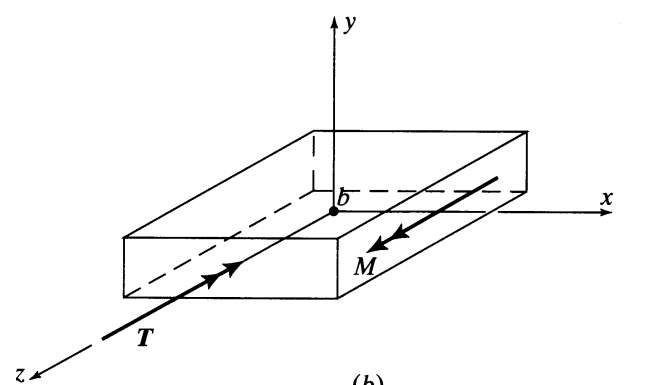

(b)

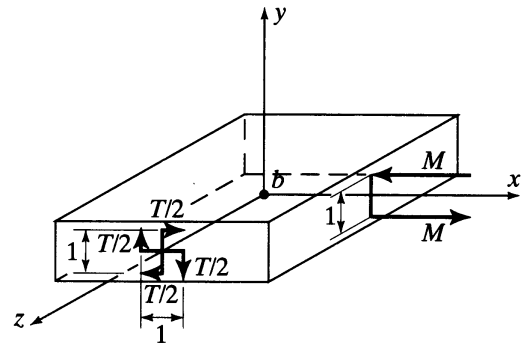

(c)

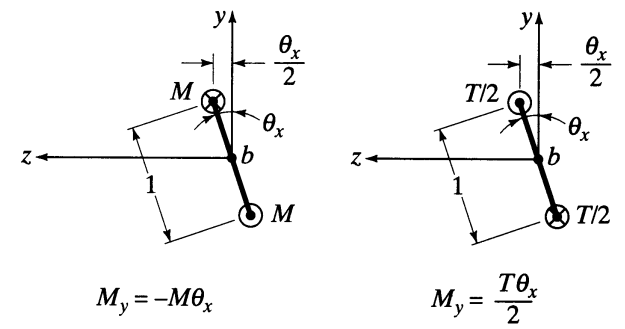

(d)

Figure 7 Loss of joint equilibrium under rotation (McGuire et al. 2000) 


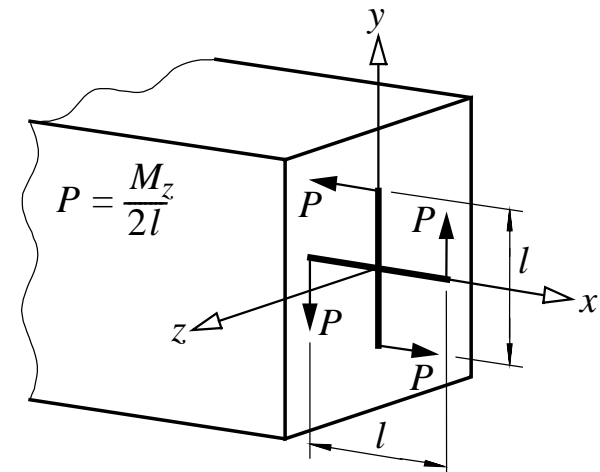

Figure 8 Semi-tangential bending moment 


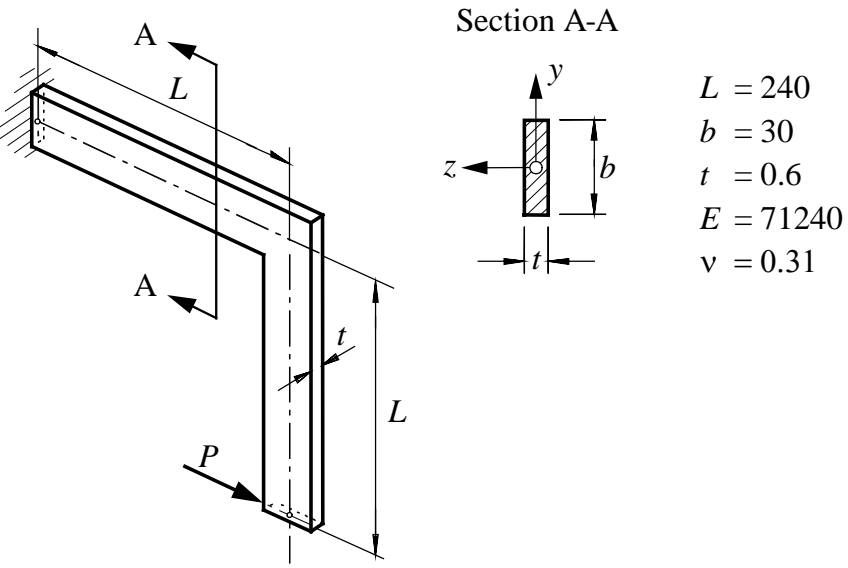

Figure 9 Clamped angle frame under a transverse tip load (Argyris et al 1979) 